\section{P2-23 CONTEXTUAL FACTORS ASSOCIATED WITH SMOKING AMONG BRAZILIAN ADOLESCENTS}

doi:10.1136/jech.2011.142976h.59

${ }^{1} \mathrm{~S} M$ Barreto, ${ }^{1} \mathrm{~L}$ Giatti, ${ }^{2} \mathrm{~L}$ Casado, ${ }^{4} \mathrm{~L}$ Moura, ${ }^{3} \mathrm{C}$ Crespo, ${ }^{1,4} \mathrm{D}$ Malta. ${ }^{1}$ Universidade Federal de Minas Gerais, Belo Horizonte, Minas Gerais, Brazil; ${ }^{2}$ Instituto Nacional do Cancer, Rio de Janeiro, Rio de Janeiro, Brazil; ${ }^{3}$ Instituto Brasileiro de Geografia e Estatistica, Rio de Janeiro, Rio de Janeiro, Brazil; ${ }^{4}$ Ministèrio da Saude, Brasilia, Distrito Federal, Brazil

Background Very few studies have examined the role of school, household and family contexts on youth smoking in middle-income countries.

Methods This work describes smoking exposure among 59992 high school students who took part in the Brazilian Survey of School Health and investigates contextual factors associated to regular smoking, defined as smoking cigarettes at least 1 day in past 30 days. Explaining variables were grouped into: sociodemographic characteristics, school context, household context and family rapport. Variables independently associated with smoking in each context were identified by multiple logistic regression.

Results 53\% were female, 89\% were aged 13-15 years. 24\% had already experimented cigarettes, $50 \%$ before the age of 12 years. Prevalence of regular smoking was $6.3 \%$ (95\% CI 5.87 to 6.74), with no sex variation. Smoking was not associated with mother's education or index of household assets. In the multivariable analysis, studying at private school, possibility of purchasing cigarettes at school and skipping classes without parent's consent increased the chance of smoking. In the household context, living with both parents was negatively associated with smoking, while having smoking parents and exposure to other people's smoking was positively related to smoking. In the family rapport, parental unawareness of what the adolescent was doing increased smoking, but having meals with the mother one or more days per week and parents' negative reactions to adolescent smoking were protective. Conclusion Results reinforce the roles of school, household and family contexts on youth' smoking behaviours and help to adjust public health policies aimed at adolescents.

\section{P2-24 SLEEP DURATION PATTERN AND CHRONIC DISEASES IN BRAZILIAN ADULTS (ISACAMP, 2008/09)}

doi:10.1136/jech.2011.142976h.60

M G Lima, P B Francisco, M B A Barros.* State University of Campinas, UNICAMP, Campinas, São Paulo, Brazil

Objective The aim of the present study was to assess the sleep patterns of the adult population of the city of Campinas (Brazil) according to socioeconomic and demographic variables and to chronic diseases and symptoms.

Methods A population-based cross-sectional study was conducted using data from the Campinas Health Survey (ISACAMP-2008-09) carried out in 2008 and 2009. A total of 2637 individuals aged 18 years or more, obtained from a probabilistic sample, were analysed. Associations between sleep pattern and the independent variables were determined using the $\chi^{2}$ test. Multinomial logistic regression models were used to adjust for confounders.

Results The prevalence of six or fewer hours of sleep was greater among individuals aged 40 years or older and among divorced. The prevalence of sleeping nine or more hours was higher among those with $<40$ years of age, divorced or single, with the lower level of schooling, who did not work and among housewives. The prevalence of short and long sleep duration was greater among individuals with heart disease, vascular problems, rheumatism/arthritis/ arthrosis, osteoporosis or emotional problems. The prevalence of short sleep duration was greater among individuals with back problems and those with three or more health conditions. There was a strong relation between sleep duration and sleep quality.

Conclusions Socio-demographic factors and the presence of health diseases were associated to sleep duration and this question should be considered in the health promotion concerns.

\section{P2-25 TRENDS AND SOCIAL INEQUALITIES ON CHRONIC DISEASES IN BRAZILIAN POPULATION: PNAD, 2003-2008}

doi:10.1136/Jech.2011.142976h.6

${ }^{1} \mathrm{M}$ B de Azevedo Barros, ${ }^{*}$ P M B Francisco, ${ }^{1} \mathrm{~L}$ M Zanchetta, ${ }^{2} \mathrm{C}$ L G Cesar. ${ }^{1}$ State University of Campinas - Medical School, Campinas, São Paulo, São Paulo, Brazil; ${ }^{2}$ University of São Paulo - Faculty of Public Health, São Paulo, São Paulo, Brazil

Introduction National Health Surveys provide essential data to monitoring health conditions and the magnitude of health inequalities. The aims of this study were to evaluate the 2008 prevalence of chronic diseases in Brazilian population comparing to 2003 data and to measure the social inequalities on the prevalence of the surveyed diseases according to educational strata.

Methods Data were obtained from the Brazilian National Survey (PNAD - health supplement) carried out on 2003 (sample of 384764 individuals) and 2008 (sample size of 391868). Prevalence and adjusted prevalence ratios were estimated using Poisson Multiple Regression with svy commands of Stata v.11.

Results The prevalence of at least one disease was significantly higher in: elders, women, low schooling level, black or indigenous people, urban residents, migrants and people living in the south region of Brazil. The most frequent diseases were: back and spinal cord disorders (13.5\% considering all age groups), hypertension $(14.0 \%)$, arthritis (5.7\%) and depression (4.1\%). Between 2003 and 2008 it was observed a significantly increase on the prevalence of diabetes, hypertension, cancer and cirrhoses, and a reduction on prevalence of chronic kidney failure and tuberculosis. All the 12 analysed diseases, with the exception of cancer and tendinitis/ tenossinovitis, showed significant higher prevalence on low educational level strata. The larger social inequalities were observed for chronic kidney failure $(P R=2.11)$, cirrhoses $(P R=2.74)$, tuberculosis $(\mathrm{PR}=1.74)$ and arthritis/rheumatism $(\mathrm{PR}=1.51)$.

Conclusion The pattern of chronic conditions is changing in the Brazilian population but the health social inequalities persist as an important national challenge.

\section{P2-26 SMOKING AND MORTALITY IN OLD AGE: 12-YEAR FOLLOW- UP OF 7000 MEN IN THE WHITEHALL RESURVEY}

doi:10.1136/jech.2011.142976h.62

${ }^{1} \mathrm{~N}$ Bhala, ${ }^{*}{ }^{1} \mathrm{~J}$ Emberson, ${ }^{2} \mathrm{M}$ Shipley, ${ }^{3} \mathrm{D}$ Leon, ${ }^{1} \mathrm{R}$ Clarke. ${ }^{1}$ Clinical Trial Service Unit 8 Epidemiological Studies Unit (CTSU), University of Oxford, Oxford, UK; ${ }^{2}$ Department of Epidemiology and Public Health, University College London, London, UK; ${ }^{3}$ London School of Hygiene \& Tropical Medicine (LSHTM), London, UK

Introduction We sought to assess the strength of associations of smoking with cause-specific mortality in older men.

Methods In a prospective study of surviving participants of the Whitehall study of male civil servants recruited in 1967-1970, 7044 were resurveyed in 1997-1998 (mean age 77 years; range 66-97) and followed for cause-specific mortality. HRs in relation to smoking habits in old age were estimated using Cox proportional hazards models after adjustment for age, prior disease and last known employment grade.

Results At resurvey, 14\% of men were current-smokers, 58\% were former-smokers and 23\% were never-smokers (the remaining 5\% reported being a never-smoker in the resurvey, but not in the initial survey). During median follow-up in survivors of 12.5 years, there were 4373 deaths (72/1000/year), of which 1838 were from 
cardiovascular disease (30/1000/yr), 1044 from cancer (17/1000/yr]); 709 from respiratory (12/1000/yr); and 782 (13/1000/yr) from other causes. Overall mortality rates were higher among current-smokers compared with never-smokers (HR 1.49, 95\% CI 1.34 to 1.65), due to increased mortality from cardiovascular disease $(1.35,1.15$ to 1.58$)$, cancer (1.71, 1.39 to 2.09), and respiratory disease (2.27, 1.75 to 2.95$)$. Among smokers, mortality rates were linearly related to the number of cigarettes smoked. Former-smokers had only moderately higher overall mortality rates compared with never-smokers (HR 1.16, 1.07 to 1.25$)$, chiefly due to continued increased risks of death from cancer (1.21, 1.03 to 1.42$)$ and respiratory disease (1.60, 1.29 to 1.98$)$.

Conclusions Among men who survived into their 70 s, continuing to smoke is associated with persistent excess vascular and non-vascular mortality.

\section{P2-27 DOES SOCIAL DISADVANTAGE IN EARLIER CHILDHOOD PREDISPOSE TO ONSET OF LIMITING LONGTERM ILLNESS (LLTI)/DISABILITY IN LATER CHILDHOOD? A POPULATION BASED STUDY USING THE UK ONS LONGITUDINAL STUDY (ONSLS)}

doi:10.1136/jech.2011.142976h.63

C Blackburn, ${ }^{*}$ N Spencer, J Read. University of Warwick, Coventry, UK

Introduction There is a lack of empirical evidence on the predictors and temporal ordering of factors associated with child disability.

Objective To examine the relationship between social disadvantage in earlier childhood and the onset of LLTI/D in later childhood in the UK ONSLS.

Methods Children born between the 1981 and 1991 UK censuses who became ONSLS members and were present at the 2001 census were identified. Data were extracted on those children who didn't have LLTI/D in 1991 but did in 2001 (index) and those with no LLTI/D on either occasion (comparison). A social disadvantage index (SDI) was constructed for 1991. Logistic regression models were fitted on LLTI/D with the SDI as the independent variable of interest adjusted for age, gender, lone parenthood, and ethnicity.

Results $60000+$ children became ONSLS members between 1981 and 1991 and were present at the 1991 and 2001 censuses. 52438 in the index and comparison groups had complete data. In bivariate analysis, social disadvantage, age, gender and lone parenthood but not ethnicity were significantly associated with the onset of LLTI/D in the index group. After adjustment for confounding, the SDI showed a finely graded association with onset of LLTI/D in the index group (most disadvantaged $\mathrm{OR}=2.12$ (1.77, 2.54); disadvantaged in two domains $\mathrm{OR}=1.45(1.20,1.75)$; disadvantaged in one domain $\mathrm{OR}=1.14(0.94,1.40))$.

Conclusions Social disadvantage in earlier childhood as a predisposing factor for the development of LLTI/D in later childhood and adolescence. Social disadvantage may be both cause and consequence of childhood LLTI/D.

\section{P2-28 FROM PROTOCOL TO PROGRESS: ESTABLISHING A REGISTRY OF CHILDREN AND YOUNG PEOPLE WITH DIABETES IN NORTH EAST ENGLAND AND NORTH CUMBRIA}

doi:10.1136/jech.2011.142976h.64

${ }^{1} \mathrm{~K}$ Blakey, ${ }^{*}{ }^{2} \mathrm{G}$ Johnson, ${ }^{1} \mathrm{R}$ McNally, ${ }^{1} \mathrm{~S}$ Court, ${ }^{3} \mathrm{~T}$ Cheetham. ${ }^{1}$ Institute of Health \& Society, Newcastle University, Newcastle Upon Tyne, UK; ${ }^{2}$ NHS Diabetes, Newcastle Upon Tyne, UK; ${ }^{3}$ Institute of Human Genetics, Newcastle University, Newcastle Upon Tyne, UK

Introduction The changing nature of diabetes in the young has underlined the importance of reliable data on where and how patients with diabetes present and live. In the former northern health region of England, an area encompassing North East England and North Cumbria, intriguing patterns in incidence and relationships with socio-economic status have been identified. The study aimed to establish a diabetes registry for children and young people in the former northern health region of England.

Methods NHS Diabetes provided regional paediatric networks with financial support to enable delivery of national outcomes and improve diabetes care in the young. During regional meetings, the North East Clinical Paediatric Network discussed a proposal to fund establishment of a regional registry for patients with diabetes aged $<18$ years. The plan was universally supported. Funding was used to employ a registry coordinator. All regulatory approval applications were completed within a rigorous and transparent ethical framework. User group involvement formed a cornerstone of the process; their feedback underpinned the production of all patient, parents and care givers documentation. A robust data security and protection policy was developed.

Results There has been significant learning regarding resources required to establish a diabetes registry including identification of sustainability issues. Methodologies have been documented and an algorithm has been generated. Data collection commenced in January 2011.

Conclusions Establishing a registry is a complex process requiring many legal and ethical considerations. The algorithm can be used as a generic template to initiate similar registries in other geographical areas or for other diseases types.

\section{P2-29 ASSOCIATION BETWEEN SELF-REPORTED DEPRESSION AND CHRONIC DISEASES: FINDINGS FROM A POPULATION BASED STUDY}

doi:10.1136/jech.2011.142976h.65

A Boing, ${ }^{*}$ G Mello, A Boing, R Moretti-Pires, K Peres, M Peres. Federal University of Santa Catarina (UFSC), Florianópilis, Santa Catarina, Brazil

Introduction Cross-sectional studies have reported associations between depression and chronic diseases, however it is necessary adjust the analysis for potential confounders. The aim of this study was to examine the association between depression and chronic diseases among adults after controlling for potential confounders.

Methods This is a population-based cross-sectional study with a sample of 1720 adults aged 20-59 years from Florianópolis, Brazil. Interviews and anthropometric measures were performed at adults' households in 2009 and included data about socio demographic characteristics, 11 self-reported chronic diseases, use of health services and physical activity at leisure time. Poisson regression models were carried out to estimate Prevalence Ratios (PR) for the association between depression and chronic diseases (none; one; two or more chronic diseases) after controlling for sex, age, marital status, income, physical activity at leisure time and hospitalisation in the last 12 months.

Results The prevalence of depression was $17.1 \%$ (95\% CI $14.9 \%$ to $19.2 \%$ ). Even after adjusting for potential confounders the association between depression and chronic diseases remained statistically significant. Those with one chronic disease presented a prevalence of depression 40\% (PR 1.4; 95\% CI 1.05 to 1.87) higher than people without disease; the prevalence of depression was $120 \%$ (PR 2.2; $95 \%$ CI 1.70 to 2.87) higher among those with two or more chronic diseases. Conclusion There is an association between depression and chronic diseases regardless potential confounders.

\section{P2-30 CHRONIC ILLNESS AND SUBJECTIVE WELL-BEING OF FAMILY MEMBERS}

doi:10.1136/jech.2011.142976h.66

C Booker, ${ }^{*}$ A Sacker. University of Essex, Colchester, Essex, UK

Background The hedonic definition of subjective well-being includes subjective perceptions of moods such as happiness and cognitive 